\title{
Grau de aceitação de mini-implantes por pacientes em tratamento ortodôntico - estudo preliminar
}

Larissa Bustamante Capucho Brandão*, José Nelson Mucha**

\begin{abstract}
Resumo
Objetivos: os mini-implantes atualmente representam um grande avanço na Ortodontia, por proporcionarem máxima ancoragem com o mínimo de cooperação dos pacientes. No entanto, ainda existem algumas dúvidas quanto ao uso destes dispositivos temporários de ancoragem no que se refere, principalmente, aos aspectos psicológicos de aceitação pelos pacientes durante o tratamento ortodôntico. Metodologia: dez pacientes adultos, portadores de má oclusão de Classe I, com biprotrusão, que foram submetidos a tratamento ortodôntico com a colocação de quatro mini-implantes nos arcos dentários, entre os primeiros molares e segundos prémolares superiores e inferiores, responderam a um questionário, com o objetivo de avaliar as condições de aceitação dos mini-implantes. Resultados: as respostas indicaram que a maioria dos pacientes aceitou prontamente o procedimento, estavam satisfeitos e recomendariam para outros pacientes (90\%), enquanto 50\% tiveram alguma preocupação com os procedimentos cirúrgicos e os outros 50\% não relataram nenhum desconforto. O tempo médio de tolerância a partir da colocação foi de 3 dias e a maioria tolerou os implantes durante todo o tratamento ortodôntico. Conclusões: pôde-se concluir que os mini-implantes foram aceitos de forma positiva pela maioria dos pacientes submetidos ao tratamento ortodôntico.
\end{abstract}

Palavras-chave: Ortodontia. Mini-implantes. Aceitação dos pacientes.

\section{INTRODUÇÃO}

$\mathrm{O}$ uso dos mini-implantes como ancoragem ortodôntica tem, atualmente, alcançado popularidade $^{1,2,3,10}$, pois eles proporcionam ancoragem absoluta em situações de movimentação ortodôntica que necessitam de máximo controle ${ }^{11,20,21,26}$. Os mini-implantes podem ser usados tanto como unidades de ancoragem direta (com forças clínicas aplicadas aos dispositivos), quanto como unidades de ancoragem indireta (com forças aplicadas às unidades dentárias que estão estabilizadas pelos mini-implantes) $)^{4,5,9}$.

Quanto à localização para a sua implantação, os mini-implantes podem ser inseridos na região sagital mediana ou paramediana do palato duro da maxila, na cortical ou osso alveolar da área molar mandibular, bicorticalmente na área molar e de pré-molar, e no osso zigomático para correções ortodônticas e ortopédicas ${ }^{12,20,25}$. A preocupação principal, em relação aos tecidos moles, é que os

* Especialista em Ortodontia pela UFF, Niterói/RJ

** Doutor em Odontologia UFRJ. Professor Titular de Ortodontia da UFF, Niterói/RJ. 
dispositivos sejam colocados em região com adequada quantidade de gengiva inserida ${ }^{6,15}$.

A altura óssea, a espessura da cortical, as estruturas anatômicas na região $0^{23,24}$ e os objetivos mecânicos irão determinar a forma, o comprimento e a espessura dos mini-implantes ${ }^{12,15,22,26}$. Apesar de serem dispositivos temporários de ancoragem ortodôntica, eles podem permanecer no local da implantação durante todo o tempo do tratamento e sua remoção é simples e rápida ${ }^{16-19}$. Mesmo com o avanço dos mini-implantes, eles ainda estão sendo usados de forma restrita, principalmente devido ao custo cirúrgico e apreensão por parte dos pacientes quanto à aceitação e tolerância aos dispositivos ${ }^{7,8,14}$. Não há, na literatura, relatos dos aspectos psicológicos e da aceitação dos pacientes em tratamentos com uso dos mini-implantes.

Diante destas últimas dúvidas, este estudo tem por objetivos: (1) verificar o grau de aceitação e satisfação dos pacientes quanto ao uso dos mini-implantes durante o tratamento ortodôntico, (2) contribuir para a melhora da resposta psicológica dos pacientes a estes novos dispositivos de ancoragem temporária e (3) esclarecer dentistas e futuros pacientes sobre a aceitação deste tipo de alternativa de ancoragem.

\section{MATERIAL E MÉTODOS}

Para a realização desta pesquisa, foram selecionados dez pacientes adultos submetidos a tratamento ortodôntico e que apresentavam como requisitos de inclusão: má oclusão de Classe I (Angle) com biprotrusão; falta de espaços para a disposição adequada de todos os dentes nos arcos dentários; perfil facial convexo, sendo esta uma das principais queixas dos pacientes; necessidade de extrações de quatro primeiros pré-molares; retração dos dentes anteriores com o máximo controle de ancoragem; planejamento de instalação de 4 mini-implantes, 2 em cada arco, entre primeiros molares e segundos pré-molares (Fig. 1).

Todos os pacientes responderam a um questionário com 12 perguntas e respostas fechadas (múl-

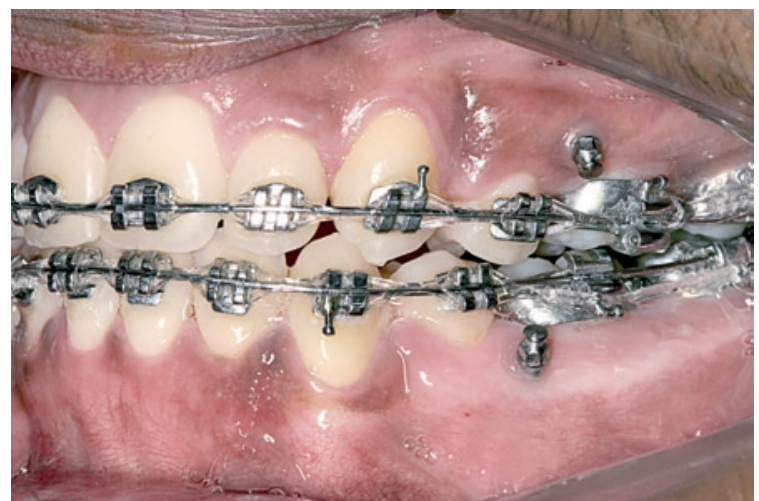

FIGURA 1 - Ilustração da localização dos mini-implantes, na maxila e mandíbula, em um paciente participante da pesquisa, após a retração dos dentes anteriores.

tipla escolha), elaborado especialmente para avaliar a aceitação, incluindo-se a adaptabilidade, efeitos colaterais, desconforto e sensibilidade dolorosa e tolerância aos mini-implantes instalados com propósito de ancoragem ortodôntica para retração dos dentes anteriores superiores e inferiores.

Os pacientes, ao serem aceitos para tratamento na Clínica de Ortodontia do Curso de Especialização em Ortodontia da Faculdade de Odontologia da UFF, assinaram termo de consentimento, de acordo com as normas de Bioética, bem como de consentimento e aceitação do plano de tratamento, com a condição de que teriam implantados quatro mini-implantes, 2 em cada arco, totalizando 40 mini-implantes.

Em todos os pacientes foram utilizados miniimplantes do tipo 994109 Ortoimplante Básicos, 1,5mm x 9mm (Conexão, Arujá/SP). As cirurgias para colocação dos mini-implantes foram realizadas pelo mesmo profissional, devidamente treinado, no Curso de Especialização em Implantodontia da Faculdade de Odontologia da UFF, sendo que a técnica cirúrgica foi semelhante para todos os pacientes.

O questionário, distribuído aos pacientes do estudo, com 12 perguntas e respostas fechadas, contendo percentagens das respostas, pode ser visto na figura 2. 


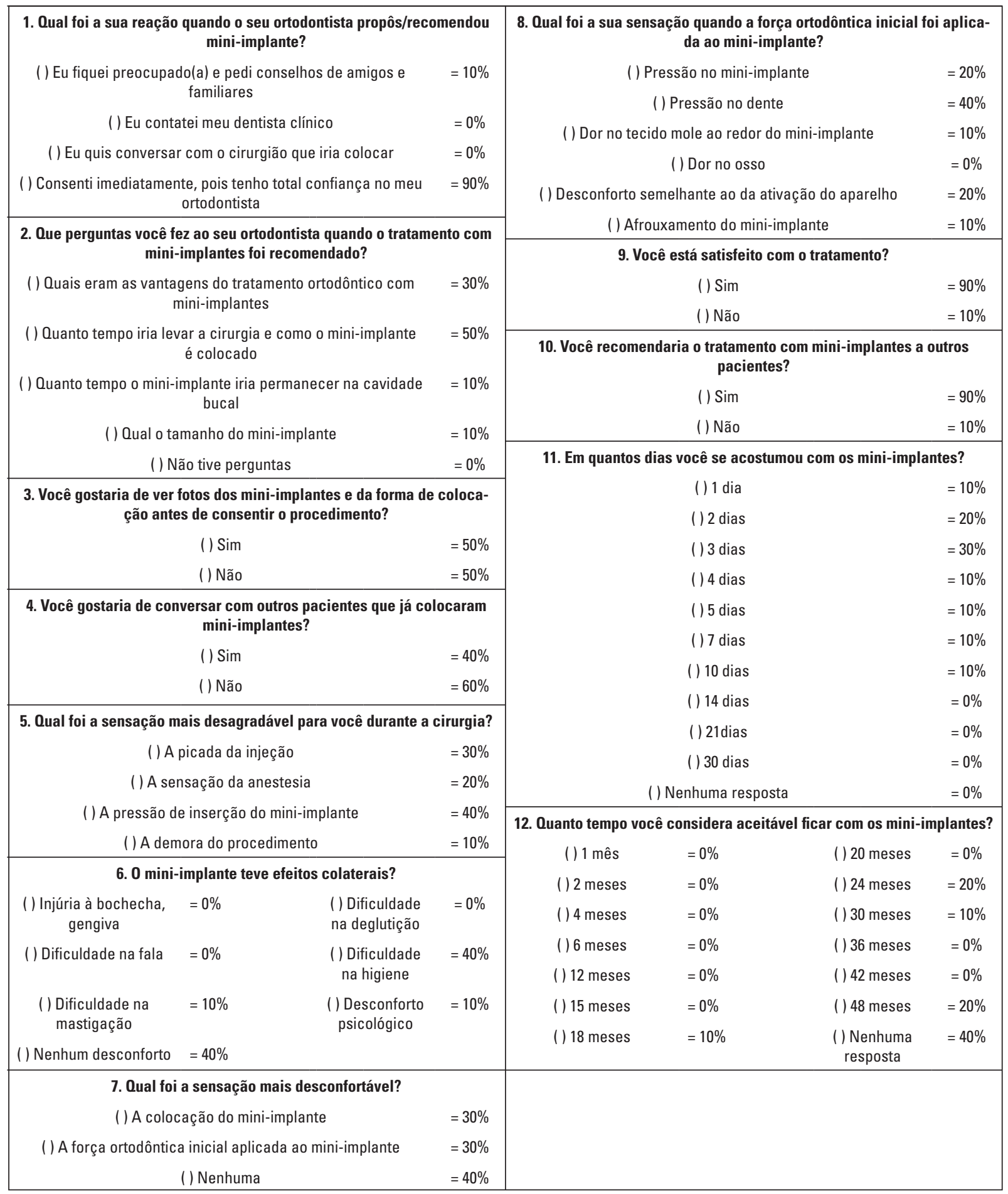

FIGURA 2 - Questionário respondido pelos pacientes submetidos a tratamento ortodôntico com colocação de 4 mini-implantes como recurso de ancoragem, para retração dos dentes anteriores superiores e inferiores. Do lado direito de cada pergunta constam os resultados, em percentuais. 


\section{RESULTADOS}

Embora 90\% dos pacientes tenham demonstrado total confiança em seus ortodontistas, aceitando as recomendações para a realização do tratamento com mini-implantes, a maioria sentiu necessidade de maiores informações, entre estas, 50\% quiseram saber quanto tempo iria levar a cirurgia e como o mini-implante seria colocado. Do total, 30\% perguntaram quais seriam as vantagens na utilização destes dispositivos, enquanto $10 \%$ gostariam de saber quanto tempo o mini-implante iria permanecer na cavidade bucal e 10\% questionaram qual o tamanho do mini-implante (Fig. 2).

As respostas em relação à visualização do tipo de dispositivo temporário de ancoragem e da forma de colocação antes do consentimento apresentaram opiniões divididas: 50\% desejaram conhecer o mini-implante e a forma de instalação, contra outros $50 \%$ que não estavam preocupados com estes aspectos. $\mathrm{O}$ mesmo pode-se dizer em relação à troca de informações com outros pacientes que passaram pelo mesmo procedimento, com 40\% que gostariam de conversar com outros pacientes, contra $60 \%$ que não viram esta conversa como um requisito para o consentimento de colocação dos mini-implantes.

Com relação à sensação mais desagradável experimentada com os procedimentos relativos à colocação dos mini-implantes, 30\% relataram a picada da agulha, 20\% a sensação da anestesia, $40 \%$ a pressão de inserção do mini-implante e 10\% a demora nos procedimentos. Considerando-se que a cirurgia é praticamente indolor, os aspectos psicológicos relacionados à anestesia e à picada foram os mais relevantes, e os demais se devem à apreensão de como seria colocado o implante (40\%) e à demora, revelando certa ansiedade com os procedimentos pouco conhecidos.

Apesar de 40\% dos indivíduos terem apresentado dificuldade na higiene, o desconforto psicológico (10\%) e na mastigação (10\%) foi mínimo. Sendo que $40 \%$ dos pacientes não relataram qualquer incômodo ou intolerância.
Considerando-se o maior desconforto, a colocação do mini-implante foi a principal queixa de 30\% dos participantes. Outros 30\% relataram a força inicial aplicada, enquanto a maioria (40\%) não relatou maiores desconfortos, tanto na colocação dos mini-implantes como na aplicação da força ortodôntica.

Após a aplicação da carga, 40\% sentiram pressão no dente, $20 \%$ no mini-implante, $20 \%$ desconforto semelhante ao da ativação do aparelho e $10 \%$ sentiram dor nos tecidos moles, ao redor do mini-implante, e deslocamento dos dispositivos de ancoragem temporária. É imprescindivel que os mini-implantes sejam colocados em faixa de gengiva inserida e os recursos para ativação (molas ou elásticos) estejam fora das áreas de lesão dos tecidos moles.

Praticamente todos os indivíduos (90\%) estavam absolutamente satisfeitos com o tipo de tratamento e recomendariam o procedimento. A maioria dos pacientes precisou de apenas 3 dias para se acostumar com os mini-implantes, o que está de acordo com o tempo de adaptação de grande parte dos procedimentos realizados em Ortodontia. Nesta amostra, 20\% e 40\% dos indivíduos revelaram que tolerariam os mini-implantes durante todo o tratamento ortodôntico.

$\mathrm{Na}$ tabela 1 são apresentadas as perguntas e as respostas com a maior percentagem em relação à aceitação dos mini-implantes pelos pacientes.

\section{DISCUSSÃO}

O questionário elaborado para avaliar o grau de aceitação dos mini-implantes, pelos pacientes submetidos a tratamento ortodôntico com estes dispositivos, mostrou-se muito útil. $\mathrm{O}$ objetivo foi avaliar a aceitação dos dispositivos temporários de ancoragem, a adaptabilidade dos pacientes aos mesmos, os possíveis efeitos colaterais, o desconforto e a sensibilidade dolorosa, bem como a tolerância durante o tempo do tratamento ortodôntico. Apesar da pequena amostra, as respostas forneceram subsídios para futuras intervenções e 
proposições de tratamentos.

A avaliação dos dados obtidos das respostas do questionário mostrou que $90 \%$ dos indivíduos confiavam em seus ortodontistas e aceitaram imediatamente o tratamento com mini-implante, no entanto, familiares e amigos ainda desempenharam um importante papel na decisão. Quando uma cirurgia, mesmo que pequena, é associada com o tratamento ortodôntico, é absolutamente normal que os pacientes apresentem certas angústias. Alguns indivíduos (10\%) necessitaram de tempo para discutir dúvidas com familiares ou amigos. Os pacientes, em geral, preferiram discutir o assunto com o ortodontista, independente da presença do cirurgião. Muito embora, alguns ortodontistas consideram que eles próprios possam implantar os dispositivos de ancoragem temporária.

Quando a participação do cirurgião se faz necessária para a colocação dos mini-implantes, considera-se importante que o plano de tratamento seja discutido primeiro com o cirurgião e posteriormente o ortodontista converse com o paciente.

$\mathrm{Na}$ consulta em que foram propostos os miniimplantes, muitos pacientes estavam interessados em saber as vantagens do tratamento ortodôntico com estes novos dispositivos de ancoragem (30\%); qual técnica cirúrgica seria usada e quanto tempo iria durar o procedimento (50\%), e o tamanho do mini-implante (40\%). O surpreendente é que o tempo de permanência com os mini-implantes $(10 \%)$ não foi a primeira preocupação. Da mesma forma, 20\% dos pacientes não tiveram perguntas em relação aos dispositivos, quando estes foram propostos. Esta percentagem se deve, provavelmente, ao fato destes pacientes terem sido selecionados na clínica do curso de pós-graduação em Ortodontia de uma instituição pública. Talvez, estes indivíduos consideraram que a não aceitação ou discordância poderiam ser fatores de cancelamento da inscrição para tratamento ortodôntico. Esta poderia ser uma tendência não controlada no presente estudo.

Tabela 1 - Perguntas e as respostas com a maior percentagem em relação à aceitação dos mini-implantes.

\begin{tabular}{|c|c|c|c|}
\hline perguntas & respostas & $\mathbf{n}$ & $\%$ \\
\hline $\begin{array}{l}\text { 1. Qual foi a sua reação quando o seu ortodontista propôs/recomendou } \\
\text { mini-implante? }\end{array}$ & $\begin{array}{l}\text { consenti imediatamente, pois tenho total } \\
\text { confiança no meu ortodontista }\end{array}$ & 9 & $90 \%$ \\
\hline $\begin{array}{l}\text { 2. Que perguntas você fez ao seu ortodontista quando o tratamento com } \\
\text { mini-implantes foi recomendado? }\end{array}$ & $\begin{array}{l}\text { quanto tempo iria levar a cirurgia e como } \\
\text { o mini-implante é colocado }\end{array}$ & 5 & $50 \%$ \\
\hline $\begin{array}{l}\text { 3. Você gostaria de ver fotos dos mini-implantes e da forma de colocação } \\
\text { antes de consentir o procedimento? }\end{array}$ & $\begin{array}{l}\operatorname{sim} \\
\text { não }\end{array}$ & $\begin{array}{l}5 \\
5\end{array}$ & $\begin{array}{l}50 \% \\
50 \%\end{array}$ \\
\hline $\begin{array}{l}\text { 4. Você gostaria de conversar com outros pacientes que já colocaram } \\
\text { mini-implantes? }\end{array}$ & não & 6 & $60 \%$ \\
\hline 5. Qual foi a sensação mais desagradável para você durante a cirurgia? & a pressão de inserção do mini-implante & 4 & $40 \%$ \\
\hline 6. 0 mini-implante teve efeitos colaterais? & $\begin{array}{l}\text { dificuldade na higiene } \\
\text { nenhum desconforto }\end{array}$ & $\begin{array}{l}4 \\
4\end{array}$ & $\begin{array}{l}40 \% \\
40 \%\end{array}$ \\
\hline 7. Qual foi a sensação mais desconfortável? & nenhuma & 4 & $40 \%$ \\
\hline $\begin{array}{l}\text { 8. Qual foi a sua sensação quando a força ortodôntica inicial foi aplicada } \\
\text { ao mini-implante? }\end{array}$ & pressão no dente & 4 & $40 \%$ \\
\hline 9. Você está satisfeito com o tratamento? & $\operatorname{sim}$ & 9 & $90 \%$ \\
\hline $\begin{array}{l}\text { 10. Você recomendaria o tratamento com mini-implantes a outros pacien- } \\
\text { tes? }\end{array}$ & $\operatorname{sim}$ & 9 & $90 \%$ \\
\hline 11. Em quantos dias você se acostumou com os mini-implantes? & 3 dias & 3 & $30 \%$ \\
\hline 12. Quanto tempo você considera aceitável ficar com os mini-implantes? & nenhuma resposta & 4 & $40 \%$ \\
\hline
\end{tabular}


Quanto à pergunta se gostariam de ver fotos dos mini-implantes e da forma de colocação antes de consentir o procedimento, as respostas foram, respectivamente, $50 \%$ sim e $50 \%$ não. Da mesma forma que a consideração anterior, os $50 \%$ que responderam não estariam mais preocupados com a solução dos seus problemas ortodônticos, que envolveria extrações dentárias de quatro primeiros pré-molares, do que com o procedimento de colocação dos mini-implantes, que seria apenas mais um fator para agilizar e otimizar os resultados. Por outro lado, os 50\% que gostariam de ver fotos e a forma de colocação estavam preocupados com este novo recurso de ancoragem, pois toda situação não usual e nova desperta curiosidade, apreensão e interesse.

Quanto ao interesse em conversar com outros pacientes que já colocaram mini-implantes, o sim de $40 \%$ dos participantes da pesquisa evidencia a troca de experiências e o conforto de pacientes em igual situação, como um fator psicológico importante para que novas técnicas e procedimentos sejam instituídos. A troca de informações entre os pacientes em uma sala de espera de consultório particular ou clínica de faculdade é fundamental para que eles adquiram confiança nos procedimentos propostos. Os $60 \%$ que aceitaram o procedimento se enquadram nos que confiam no ortodontista ou até já possuem algumas informações a respeito.

Com relação à pergunta 5 , sobre qual a sensação mais desagradável durante a cirurgia, a picada da injeção foi a resposta de $30 \%$ dos pacientes, a sensação da anestesia foi a resposta de $20 \%$, a pressão de inserção do mini-implante de $40 \%$ e a demora do procedimento de $10 \%$. A percentagem de $40 \%$ que relatou o maior desconforto da pressão no mini-implante é compreensível, pois trata-se de algo novo, desconhecido e que foi proposto pelo ortodontista para a melhor solução do problema. Mesmo com a aceitação do procedimento, a apreensão psicológica de ter um "parafuso inserido no osso" causa alguma preocu- pação e desconforto psicológico, mesmo que não haja relato de dor.

Ao ser feita a pergunta se os mini-implantes tiveram efeitos colaterais, a dificuldade na higiene foi a resposta de $40 \%$ dos participantes da pesquisa, a dificuldade na mastigação em 10\%, desconforto psicológico em $10 \%$ e nenhum desconforto em $40 \%$. Deve-se lembrar que todos os pacientes apresentavam aparelhos fixos em ambos os arcos e dispositivos, tais como alças, elásticos ou molas, para a retração e fechamentos dos espaços das extrações. Torna-se compreensível que a dificuldade de higienização seja o fator de desconforto em 40\% dos participantes, além das exigências do ortodontista para que tenham um excelente controle de higiene, sendo este um fator do sucesso da estabilidade dos mini-implantes. Por outro lado, $40 \%$ dos participantes não relataram nenhum desconforto.

Quanto à sensação mais desconfortável, a colocação do mini-implante foi a resposta de 30\%, a força ortodôntica inicial aplicada ao mini-implante de $30 \%$ e nenhum desconforto em $40 \%$ dos pacientes. Esta percentagem está de acordo com o item anterior, em que, igualmente, $40 \%$ não relataram nenhum desconforto. As forças ortodônticas aplicadas foram em torno de 300 a 450 gramas.

Para a questão 8 , sobre qual a sensação quando a força ortodôntica inicial foi aplicada ao miniimplante, a resposta de pressão ao mini-implante foi de $20 \%$, a pressão no dente de $40 \%$, dor no tecido mole ao redor do mini-implante de $10 \%$, desconforto semelhante ao da ativação do aparelho de $20 \%$ e deslocamento do mini-implante de $10 \%$. A resposta de nenhuma dor no osso $(0 \%)$, como era de se esperar, confirma que a sensação dolorosa, ou a dor psicológica, é mais relevante do que a real, visto que o desconforto principal foi a pressão no dente (40\%), e não no osso (0\%) ou no mini-implante (20\%). Igualmente, 20\% dos participantes do estudo compararam a sensação da ativação do mini-implante à ativação do aparelho.

Em relação à pergunta: "Você está satisfeito 
com o tratamento?", obteve-se resposta positiva em $90 \%$ dos pacientes, contra apenas 10\% de resposta negativa. Pode-se considerar que o descontentamento se deve à necessidade de reinstalação do mini-implante, devido a problemas técnicos na implantação. Conseqüentemente, era de se esperar que alguma insatisfação poderia estar presente nesta situação. A mesma consideração pode ser feita para a questão seguinte: "Você recomendaria o tratamento com mini-implantes a outros pacientes?". Onde 90\% responderam que sim e apenas $10 \%$ que não.

Os pacientes necessitaram de, aproximadamente, 10 dias para se acostumar ao mini-implante, com alguns (60\%) já estando totalmente adaptados nos 3 primeiros dias após a colocação, enquanto outros precisaram de um tempo maior, até 10 dias, mas não ultrapassando este tempo. Pode-se dizer, portanto, que a adaptação aos mini-implantes apresentou uma variação entre $1 \mathrm{e}$ 10 dias, com possibilidade de adaptação da maior parte dos pacientes em 3 a 4 dias. Ao contrário dos implantes tradicionais, onde se deseja a osteointegração, que ocorre em, aproximadamente, 3 a 6 meses sem aplicação de carga, com os mini-implantes a osteointegração não é desejável, por isso a aplicação da carga poderá ser imediata. O gráfico 1 ilustra o tempo de adaptação com os respectivos percentuais.

Apesar da grande contribuição destes dispositivos de ancoragem temporária, existem algumas dificuldades, pois os mesmos envolvem procedi-

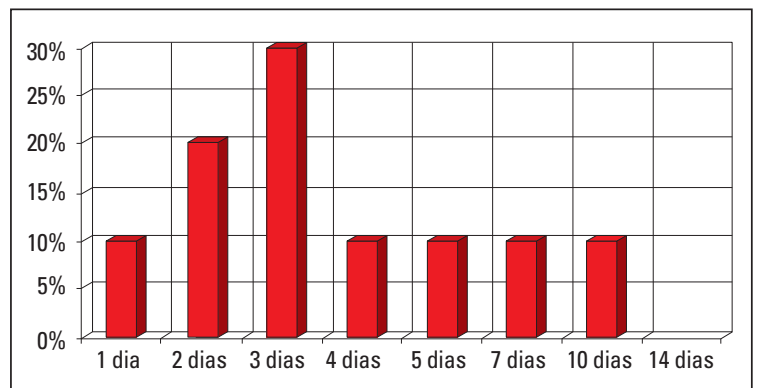

GRÁFICO 1 - Percentagem das respostas em relação ao número de dias em que os pacientes se acostumaram aos mini-implantes. mentos cirúrgicos, o custo é maior e o conforto, muitas vezes, menor (dependendo do local) em relação aos métodos de tratamento tradicionais. Portanto, deve-se esclarecer aos pacientes que estes procedimentos cirúrgicos são simples e realizados com pequena quantidade de anestésico local ou até anestésico tópico ${ }^{13}$ (e algumas vezes até sem anestesia), o tempo cirúrgico é pequeno, sendo geralmente em torno de 15 a 20 minutos. Em algumas situações, esta cirurgia poderá evitar a necessidade de tratamentos mais complexos, como cirurgias ortognáticas, onde o paciente estará sob anestesia geral. Além disso, a eficácia do tratamento será maior, em menor tempo, nos casos que requeiram maior controle de ancoragem.

Os estudos em relação ao tamanho dos miniimplantes (largura e altura) têm evoluído muito, mas o interessante é que este aspecto acabou sendo mais importante, sobre o ponto de vista mecânico e anatômico, para os profissionais envolvidos do que como preocupação por parte dos pacientes. Os pacientes só sentem a porção supramucosa do miniimplante, cuja cabeça, dependendo do caso, poderá ser recoberta com uma resina flow, para evitar desconforto. Enquanto metade dos pacientes não relatou nenhum efeito colateral, 20\% relataram incômodo na mastigação e desconforto psicológico e 40\% apresentaram dificuldade na higiene.

Pode-se considerar que este procedimento é altamente promissor, em relação à aceitação pelos pacientes, pois $90 \%$ estavam satisfeitos com o tratamento e recomendariam o tratamento a outros pacientes.

Considerando-se de 24 a 30 meses como a média de tempo aceitável para os tratamentos ortodônticos, 50\% disseram tolerar este tempo de tratamento com os mini-implantes e $40 \%$ não puderam julgar quanto tempo tolerariam os dispositivos de ancoragem temporária, significando que eles suportariam ainda mais tempo.

Todos os casos tiveram sucesso em relação à mecânica empregada e estabilização dos miniimplantes para os propósitos de ancoragem, sendo 
que apenas um implante se deslocou, necessitando ser removido antecipadamente. Apesar de não ter comprometido o resultado mecânico, o fato pode ter gerado insatisfação do paciente, acarretando as respostas não tão satisfatórias em algumas perguntas $(10 \%)$.

Consideram-se estes dados provisórios, pois sugere-se e pretende-se realizar estudos em amostras maiores, para confirmar estes resultados. Porém, pode-se afirmar, de maneira geral, que a utilização destes dispositivos de ancoragem temporária foi extremamente eficaz e a aceitação pelos pacientes foi altamente significativa, constituindo-se, portanto, em uma ferramenta auxiliar ao alcance do ortodontista, para casos bem selecionados e que requeiram controle máximo de ancoragem.

\section{CONCLUSÕES}

Baseado nas respostas dos pacientes ao questionário, pode-se concluir que:

1- A percentagem de pacientes satisfeitos com os mini-implantes foi de $90 \%$;

2- As maiores preocupações dos pacientes, ao serem propostos os mini-implantes, foram: em relação ao tempo da cirurgia e como são colocados (50\%), quais as vantagens (30\%) e qual o tamanho (10\%). Sendo que $20 \%$ não revelaram preocupações;

3- Quanto à visualização dos mini-implantes e necessidade de conversar com outros pacientes em relação ao procedimento, apenas metade da amos- tra mostrou-se interessada, sendo que os demais não necessitaram de maiores esclarecimentos;

4- Na colocação dos mini-implantes as sensações mais desagradáveis, em ordem decrescente, foram: a inserção do mini-implante (40\%); a picada da injeção (30\%); a sensação da anestesia (20\%); e a demora do procedimento (10\%);

5- Após a implantação, 40\% não relataram nenhum desconforto, sendo que a maior dificuldade foi durante a higienização (40\%), mastigação $(10 \%)$ e alguma apreensão psicológica (10\%);

6- Quanto à sensação mais desconfortável, 30\% relataram a colocação do mini-implante, $30 \%$ a força ortodôntica e $40 \%$ toleraram bem sem queixas de desconforto;

7- Quando à sensação da aplicação da força, $40 \%$ relataram pressão no dente, $20 \%$ pressão no mini-implante, $20 \%$ pressão como a da ativação do aparelho, pouca dor foi sentida no tecido mole ao redor do mini-implante (10\%), sensação de afrouxamento $(10 \%)$ e nenhuma dor foi sentida no osso adjacente $(0 \%)$;

8- Do total, $90 \%$ estavam satisfeitos com o tratamento com mini-implante e o recomendariam a outros pacientes;

9- Os pacientes se acostumaram com os miniimplantes, na média, em 3 dias, com um tempo máximo de 10 dias de adaptação;

10- Os pacientes revelaram que tolerariam os mini-implantes durante todo o tratamento ortodôntico. 


\title{
The mini-implants acceptance rate by patients in orthodontic treatments - a preliminary study
}

\begin{abstract}
Objective: Actually, mini-implants as anchorage resources have been spotlight in Orthodontics, providing maximum anchorage with minimum patient's cooperation. However, there are still some restrictions regarding the use of these temporary anchorage devices, and among these, the psychological aspects and the patient's acceptance, which still not properly evaluated. Methods: Ten adults patients, with Class I biprotrusion malocclusion, that were submitted to orthodontic treatment with the implantation of four mini-implants, in the dental arches, among upper and lower first molar and second premolar teeth, in a total of 40 mini-implants, answered a questionnaire elaborated to evaluate the acceptance conditions of mini-implants by this patients. Results: The answers obtained from the questionnaires were transformed in percentage and indicated that: most patients accepted quickly the procedure (90\%); with some preoccupation with the surgical procedures (50\%); $50 \%$ related no discomfort; on average they tolerated the mini-implants in 3 days; $90 \%$ were satisfied with the treatment; and $90 \%$ would recommend the treatment to others patients. Most patients tolerated mini-implant during all time of the orthodontic treatment. Conclusions: According to the results obtained with the present evaluation, it can be concluded that the mini-implants acceptance was very prominent by the patients. Larger samples' studies are necessary.
\end{abstract}

Key words: Orthodontics. Mini-implants. Patient's acceptance.

\section{REFERÊNCIAS}

1. ARAÚJO, T. M.; NASCIMENTO, M. H. A.; BEZERRA, F. SOBRAL, M. C. Ancoragem esquelética em Ortodontia com miniimplantes. Rev. Dental Press Ortodon. Ortop. Facial, Maringá, v. 11, n. 4, p. 126-156, jul./ago. 2006

2. $\mathrm{BAE}, \mathrm{S}$. M. et al. Clinical application of micro-implant anchorage. J. Clin. Orthod., Boulder, v. 36, no. 5, p. 298-302, May 2002.

3. BLOCK, M. S.; HOFFMAN, D. R. A new device for absolute anchorage for Orthodontics. Am. J. Orthod. Dentofacial Orthop., St. Louis, v. 107, no. 3, p. 251-258, Mar. 1995.

4. CELENZA, F.; HOCHMAN, M. N. Absolute anchorage in Orthodontics: direct and indirect implant-assisted modalities. J. Clin. Orthod., Boulder, v. 34, no. 7, p. 397-402, July 2000.

5. $\mathrm{CHO}, \mathrm{H}$. J. Clinical applications of mini-implants as orthodontic anchorage and the peri-implant tissue reaction upon loading. J. Calif. Dent. Assoc., San Francisco, v. 34, no. 10, p. 813-820, Oct. 2006.

6. COSTA, A.; PASTA, G.; BERGAMASCHI, G. Intraoral hard and soft tissue depths for temporary anchorage devices. Semin. Orthod., Philadelphia, v. 11, no. 1, p. 10-15, 2005.

7. FREUNDENTHALER, J. W.; HAAS, R.; BANTLEON, H. P. Bicortical titanium screws for critical orthodontic anchorage in the mandible: a preliminary report on clinical applications. Clin. Oral Implants Res., Copenhagen, v. 12, no. 4, p. 358-363, Aug. 2001

8. GÜNDÜZ, E.; SAVIO, T. T. S. D.; KUCHER, G.; SCHNEIDER, B.; BANTLEON, H. P. Acceptance rate of palatal implants: a questionnaire study. Am. J. Orthod. Dentofacial Orthop., St. Louis, v. 126, no. 5, p. 623-626, Nov. 2004

9. HERMAN, R. J.; CURRIER, G. F.; MIYAKE, A. Mini-implant anchorage for maxillary canine retraction: a pilot study. Am. J. Orthod. Dentofacial Orthop., St. Louis, v. 130, no. 2, p. 228-235, Aug. 2006.
10. KANOMI, R. Mini-implant for orthodontic anchorage. J. Clin. Orthod., Boulder, v. 31, no. 11, p. 763-767, Nov. 1997

11. KEIM, R. G. Answering the questions about miniscrews. Editor's Corner. J. Clin. Orthod., Boulder, v. 39, no. 1, p. 7-8, Jan. 2005

12. KIM, T. W.; KIM, H.; LEE, S. J. Correction of deep overbite and gummy smile by using a mini-implant with a segmented wire in a growing Class II Division 2 patient. Am. J. Orthod. Dentofacial Orthop., St. Louis, v. 130, no. 5, p. 676-685, Nov. 2006.

13. KRAVITZ, N. D.; KUSNOTO, B. Placement of mini-implants with topical anesthetic. J. Clin. Orthod., Boulder, v. 40, no. 10, p. 602-604. Nov. 2006.

14. KURODA, S.; SUGAWARA, Y.; DEGUCHI, T.; KYUNG, H. M.; TAKANO-YAMAMOTO, T. Clinical use of miniscrew implants as orthodontic anchorage: Success rates and postoperative discomfort. Am. J. Orthod. Dentofacial Orthop., St. Louis, v. 131, no. 1, p. 9-15, Jan. 2007.

15. KYUNG, H. M.; PARK, H. S.; BAE, S. M.; SUNG, J. H.; KIM, I. B. Development of orthodontic micro-implants for intraoral anchorage. J. Clin. Orthod., Boulder, v. 37, n. 6, p. 321-328, June 2003.

16. LIOU, E. J.; PAI, B. C.; LIN, J. C. Do miniscrew remain stationary under orthodontic forces? Am. J. Orthod. Dentofacial Orthop., St. Louis, v. 126, no. 1, p. 42-47, July 2004.

17. MAH, J. K.; BERGSTRAND, F. Temporary anchorage devices: a status report. J. Clin. Orthod., Boulder, v. 339, no. 3, p. 132-136, Mar. 2005.

18. MELSEN, B. Mini-implants: where are we? J. Clin. Orthod., Boulder, v. 39, no. 9, p. 539-547, Sept. 2005.

19. MIYAWAKI, S. et al. Factors associated with the stability of titanium screws placed in the posterior region for orthodontic anchorage. Am. J. Orthod. Dentofacial Orthop., St. Louis, v. 124 , no. 4, p. 373-378, Oct. 2003 
20. PARK, H. S.: BAE, S. M.: KYUNG, H. M.: SUNG, J. H. Microimplant anchorage for treatment of skeletal Class I bialveolar protrusion. J. Clin. Orthod., Boulder, v. 35, no. 7, p. 417-422, July 2001.

21. PARK, H. S. et al. Micro-implant anchorage for treatment of skeletal Class I bialveolar protrusion. J. Clin. Orthod., Boulder, v. 35, no. 7, p. 417-422, July 2001.

22. PARK, H. S.; LEE, S. K.; KWON, O. W. Group distal movement of teeth using microscrew implant anchorage. Angle Orthod. Appleton, v. 75, no. 4, p. 602-609, Apr. 2005.

23. POGGIO, P. M.; INCORVATI, C.; VELO, S.; CARANO, A. "Safe zones": a guide for miniscrew positioning in the maxillary and mandibular arch. Angle Orthod., Appleton, v. 76, no. 2, p. 191-197, Feb. 2006.
24. SCHNELLE, M. A.; BECK, F. M.; JAYNES, R. M.; HUJA, S. $S$. A radiographic evaluation of the availability of bone for placement of miniscrews. Angle Orthod., Appleton, v. 74, no. 6, p. 832-837, June 2004

25. THIRUVENKATACHARI, B. et al. Comparison and measurement of the amount of anchorage loss of the molars with and without the use of implant anchorage during canine retraction. Am. J. Orthod. Dentofacial Orthop., St. Louis, v. 129, no. 4, p. 551 554, Apr. 2006.

26. YAO, C. J. et al. Maxillary molar intrusion with fixed appliances and mini-implant anchorage studied in three dimensions. Angle Orthod., Appleton, v. 75, no. 5, p. 754-760. 2005.
Endereço para correspondência

José Nelson Mucha

Rua Visconde de Pirajá, 351 sala 814

CEP: 22.410-003 - Ipanema - Rio de Janeiro / RJ

E-mail: nelsonmucha@wnetrj.com.br 